monitoring was 11.5 hours. Continuous EEG monitoring is recommended in children with acute encephalopathy. (Greiner HM, Holland K, Leach JL, Horn PS, Hershey AD, Rose DF. Nonconvulsive status epilepticus: the encephalopathic pediatric patient. Pediatrics March 2012;129(3):e748-55). (Respond: Hansel M Greiner MD, Cincinnati Children's Hospital Medical Center, 3333 Burnet Ave, MLC 2015, Cincinnati, OH 45229. E-mail: hansel.greiner@cchmc.org).

COMMENT. Risk factors for NCSE in children include a prior clinical seizure and acute cortical imaging abnormality. When both of these variables were present, the probability of NCSE was high (82\%). If NCSE is suspected, continuous EEG monitoring is important not only in high-risk neonates in the ICU but also in children and adolescents with disturbed consciousness and symptoms of encephalopathy. The Cincinnati investigators defined NCSE as continuous 30-minute electrographic seizure activity with non-convulsive clinical symptoms or repeated briefer electrographic seizures comprising at least 30 minutes of a 1-hour period (Greiner HM et al. 2012).

A NCSE etiological classification included metabolic disorders, coma, acute cerebral lesions, and preexisting epilepsy (Maganti $R$ et al. Epilepsy Behav 2008;12(4):572-586). NCSE constitutes about 25-50\% of all cases of status epilepticus. In comatose patients, NCSE diagnosis is often difficult and potentially fatal if untreated. The EEG may show a variety of rhythmic or periodic patterns, some of unclear significance. Of 19 pediatric patients with NCSE identified from the database of the Columbia University Epilepsy Center, 6 had periodic lateralized epileptiform discharges (PLEDS), and 1 had generalized PEDS. Periodic discharges were associated with worse outcome. (Tay SK et al. Epilepsia 2006;47(9):1504-1509). The most frequent etiology of NCSE in this study was acute hypoxic-ischemic encephalopathy (26\%); other causes included metabolic (21\%), infection (16\%), AED change (16\%), refractory epilepsy $(11 \%)$, and intracranial hemorrhage (11\%). Prompt recognition of NCSE by continuous EEG monitoring should lead to early treatment and improved prognosis.

\title{
HIGH-FREQUENCY EEG OSCILLATIONS: NEW BIOMARKER IN EPILEPSY
}

Researchers at University Medical Center Utrecht, The Netherlands and other centers review the pathophysiology, clinical relevance, identification, and interpretation of high-frequency oscillations (HFOs, $>80 \mathrm{~Hz}$ ) in the epileptic EEG. HFOs are further classified in ripples $(80-250 \mathrm{~Hz})$, fast ripples $(250-600 \mathrm{~Hz})$, and very-HFOs $(>1,000 \mathrm{~Hz})$. HFOs are observed between seizures, at seizure onset, and during seizures. Interictal HFOs occur during slow-wave sleep. The intracranial EEG is sampled at $=>>2,000 \mathrm{~Hz}$. In patients with focal epilepsy who may benefit from surgery, removal of brain tissue generating HFOs results in better outcome than removal of the seizure onset zone. Interictal HFOs are reliable markers of the seizure onset zone, and better than epileptic spikes. HFOs occur in different types of epilepsy, mesiotemporal with hippocampal sclerosis and also in extratemporal epilepsies with tumors, focal cortical dysplasia, and nodular heterotopia. HFOs are sometimes recorded in nonlesional epilepsies. Evaluation of seizures with HFO recordings $>80 \mathrm{~Hz}$ may improve the pre-surgical workup and outcome and reduce the necessity for invasive monitoring. (Zijlmans M, Jiruska P, 
Zelmann R, Leijten FSS, Jeffreys JGR, Gotman J. High-frequency oscillations as a new biomarker for epilepsy. Ann Neurol February 2012;71:169-178). (Respond: Dr Zijlmans, Department of Neurology and Neurosurgery, University Medical Center Utrecht, The Netherlands. E-mail: g.j.m.zijlmans@umcutrecht.nl).

COMMENT. Methods used to determine the extent of epileptic foci of brain tissue before and during cortical resection include EEG telemetry, ECoG, MRI, histological, and immunohistochemical. Tissue markers of epileptic foci include mitochondrial "hypermetabolic" neurons and a-B-crystallin (Sarnat HB et al. Can J Neurol Sci 2011;38(6):909-17)(Sarnat HB, Flores-Sarnat L. ibid. 2009;36(5):566-74) (Pediatr Neurol Briefs 2012;26(1):5-6)(ibid. 2009;23(11);81-82). The Annals review provides a comprehensive account of the utility of HSOs as a biomarker of epileptogenesis.

HSOs are studied primarily in mesiotemporal epilepsies. In a series of 30 consecutive pediatric patients at UCLA undergoing surgery for refractory epilepsy due mainly to extratemporal lesions, ECoGs were recorded at $2,000 \mathrm{~Hz}$ and visually inspected for fast ripples (FR 250-500Hz). FR episodes were identified in ECoGs from 24 patients (80\%); FR-containing cortex was removed in 19 and all became seizure-free. FRcontaining cortex was found outside of abnormalities defined by MRI and FDG-PET in 6 children. The authors conclude that interictal fast ripples are an excellent surrogate marker of epileptogenesis. (Wu JY et al. Neurology 2010;75(19):1686-94).

\section{EEG PATTERNS AND GENOTYPES IN ANGELMAN SYNDROME}

Researchers at the Children's Hospital, Boston, other centers in the US, and Poznan University, Poland, prospectively analyzed EEGs from participants in the NIH Angelman Syndrome Natural History Study. Of 160 enrolled patients (2006-2010), 115 had complete data (58 boys, median age 3.6 years). EEG findings included intermittent rhythmic delta waves $(83.5 \%)$, interictal epileptiform discharges $(74.2 \%)$, intermittent rhythmic theta waves $(43.5 \%)$, and posterior rhythm slowing (43.5\%). Centro-occipital and centro-temporal delta waves decreased with age $(\mathrm{p}=0.01$ and 0.03$)$, and EEG patterns are age-dependent. EEG patterns and seizure types were not correlated significantly with genotypes. Using a classification tree to predict specific genotypes based on EEG features, deletions class-2 $(5.0 \mathrm{Mb})$ were associated with $>50 \%$ intermittent rhythmic theta activity, and deletions class-1 $(5.9 \mathrm{Mb})$ with $<50 \%$ intermittent rhythmic theta activity and epileptiform discharges while awake. EEG patterns are important biomarkers in Angelman syndrome and may suggest the underlying genetic etiology. (Vendrame M, Loddenkemper T, Zarowski M, et al. Analysis of EEG patterns and genotypes in patients with Angelman syndrome. Epilepsy Behav March 2012;23:261-265).(Response: Dr Sanjeev V Kothare, Children's Hospital Boston, Fegan 9, 300 Longwood Ave, Boston, MA 02115. E-mail: Sanjeev.Kothare@childrens.harvard.edu).

COMMENT. Boyd SG and colleagues at Great Ormond Street Children's Hospital, London, UK first described EEG patterns that were considered characteristic of Angelman syndrome: 1) Persistent rhythmic 4-6/s activity ( $>200 \mathrm{mcV})$ while awake; 2) prolonged runs of rhythmic $2-3 / \mathrm{sec}$ activity $(200-500 \mathrm{mcV})$ anteriorly; and 3) spikes 